\title{
Belphégor
}

\section{Mystère et crime dans le récit policier émergent : l'exemple de «The Avenger » de Thomas De Quincey}

\section{Marc Vervel}

\author{
(2) OpenEdition \\ Journals \\ Édition électronique \\ URL : https://journals.openedition.org/belphegor/4419 \\ DOI : 10.4000/belphegor.4419 \\ ISSN : 1499-7185 \\ Éditeur \\ LPCM
}

Référence électronique

Marc Vervel, « Mystère et crime dans le récit policier émergent : l'exemple de « The Avenger » de Thomas De Quincey », Belphégor [En ligne], 19-2 | 2021, mis en ligne le 04 janvier 2022, consulté le 20 septembre 2022. URL : http://journals.openedition.org/belphegor/4419; DOI : https://doi.org/ 10.4000/belphegor.4419

Ce document a été généré automatiquement le 20 septembre 2022.

\section{(c) (i) (9)}

Creative Commons - Attribution - Pas d'Utilisation Commerciale - Pas de Modification 4.0 International - CC BY-NC-ND 4.0

https://creativecommons.org/licenses/by-nc-nd/4.0/ 


\title{
Mystère et crime dans le récit policier émergent : l'exemple de "The Avenger » de Thomas De Quincey
}

\author{
Marc Vervel
}

1 Dans la première moitié du dix-neuvième siècle, alors que la thématique criminelle prend une importance inusitée ${ }^{1}$, le roman fait du questionnement herméneutique l'un de ses enjeux majeurs. Le mystère articule ce rapport à un inconnaissable et promet volontiers au lecteur une narration envisagée sous le signe du dévoilement. Le mot, dont le succès est intrinsèquement lié à la vogue gothique, s'est de fait chargé des complexités et ambivalences attachées à ses origines religieuses, pour susciter l'idée de vérités enfouies et prises dans de secrètes logiques de manipulations ${ }^{2}$. Il renvoie à un rapport essentiellement paradoxal que le roman serait à même d'entretenir avec le vrai : ce que dit le roman sous le signe du mystère, c'est qu'il va donner à connaître ce qui relève de l'interdit et de l'inconnu. Il est bien fait pour susciter la curiosité du lecteur, tout en ouvrant à l'occasion sur la nostalgie d'un sens transcendant dont le texte pourrait se faire porteur; il revêt ainsi une fonction éminemment narratologique en même temps qu'il est susceptible de pointer le cas échéant vers une éventuelle portée herméneutique du propos ${ }^{3}$.

2 Au-delà des secrets des châteaux gothiques ou des crimes et de la misère des bas-fonds, ce problème peut s'autonomiser pour concerner la manière dont le roman est susceptible de se constituer en instance de connaissance. Les stratégies traditionnelles de légitimation romanesque en lien avec le rapport au vrai sont alors à même de se voir réinvesties pour en creuser les lignes de fracture et ouvrir sur le travail de la curiosité du lecteur ${ }^{4}$. La vérité qu'il s'agirait de dire sur le mode romanesque concerne un monde caché, mais aussi les difficultés à appréhender ce qui échappe radicalement, se dérobe au sens et joue le rôle d'une menace portant sur la possibilité même de proposer une formalisation effective de la réalité. 
3 Le crime joue un rôle privilégié dans cette économie ; il est de l'ordre de la transgression, et concerne la subversion du sens et le jeu de l'exhibé et de l'enfoui - peu à peu, il n'est plus saisi du côté du criminel, mais envisagé du point de vue de l'instance qui cherche à le fixer pour en réduire l'exercice : c'est le mystère, qui doit être réduit à néant pour conjurer les menaces d'allure inquiétante et obscure. Surveiller et punir envisage ce moment d'émergence d'une nouvelle épistémè comme celui de la naissance de l'idéologie disciplinaire, et d'une disparition progressive du spectacle du châtiment du criminel $^{5}$; le roman peut alors être envisagé comme le lieu cherchant à donner à voir ce spectacle disparu et désormais impossible, mais aussi comme ce qui donne à penser le crime comme procédant à son tour d'une logique d'illisibilité et d'invisibilité. Par ce biais, le roman est en mesure de problématiser la relation du lecteur au monde envisagé comme ensemble de signes incohérents au sens fuyant, selon une stratégie qui fait déjà du texte un récit policier en puissance, une enquête sur une transgression se déroulant dans l'ombre. Pour étudier cette question, on envisagera "The Avenger " (1838) de Thomas de Quincey (1785-1859), en lien avec On Murder Considered as One of the Fine Arts (dont la première conférence a été publiée en 1827) ; avant les nouvelles de Poe consacrées au chevalier Dupin se lit déjà, ici, l'idée que le meurtre équivaut à un trouble fondamental dans le sens, susceptible d'affecter le fonctionnement même du texte. Dans cette économie, le « mystère » est alors susceptible de jouer son rôle.

4 Aux yeux de Michel Foucault, Thomas de Quincey incarne le passage de la littérature populaire du crime au roman policier moderne, associé à la conception esthétisante du dandy confronté au meurtre, où s'esquisse déjà le type romanesque dont dérivera Sherlock Holmes ${ }^{6}$. Mais l'œuvre protéiforme de De Quincey témoigne de pratiques littéraires plus diverses et complexes qu'il n'y paraît. Il a, dans sa jeunesse, lu et aimé la littérature gothique ${ }^{7}$, et s'il s'en est départi peu à peu au profit d'une approche intellectualiste et distanciée de l'écriture, il n'en a pas moins écrit plusieurs textes peu ou prou assimilables à ce genre ${ }^{8}$; il est par ailleurs un rédacteur régulier du Blackwood's Magazine, avec lequel il entretient des relations ambivalentes. La question de la façon dont il use du mystère pour jouer avec les sensations du lecteur tout autant que pour susciter sa curiosité est alors d'autant plus intéressante à envisager que De Quincey se joue volontiers des étiquettes, et aime à emprunter à diverses traditions pour construire une œuvre complexe et volontiers ambiguë ${ }^{10}$.

5 Revenons donc, pour commencer, sur le texte qui, aux yeux de Foucault, incarne l'invention d'une littérature du crime se donnant sous le signe de l'élitisme. Le célèbre et sulfureux On Murder Considered as One of the Fine Arts ${ }^{11}$ 'est ni un roman, ni un texte interrogeant le caractère énigmatique du monde. Pour autant, l'intérêt pour la dimension esthétisante du meurtre amène à y revenir en guise de préambule à l'analyse, pour voir si la question du sens insaisissable y trouve malgré tout sa part. La première des «conférences » qui constituent le texte de De Quincey a d'abord été publiée en $1827^{12}$; elle a été suivie d'une seconde conférence en 1839, avant que cellesci ne soient assorties d'un post-scriptum et publiées en volume en 1854.

6 Le texte se présente donc, on le sait, comme une suite de conférences, énoncées par un narrateur-orateur au statut incertain, peut-être assassin lui-même, et membre en tout cas de la "Société des Connaisseurs en Meurtre » ${ }^{13}$, à mi-chemin du club mondain et de la société secrète. Il assume une conception provocatrice du meurtre envisagé comme activité esthétique susceptible d'ouvrir à l'admiration désintéressée de l'amateur d'art, et se centre notamment sur la figure du célèbre assassin John Williams. Le mot 
«mystery » est absent des deux conférences, où le crime n'est pas constitué en enjeu herméneutique ; il apparaît en revanche dans le post-scriptum, qui réintroduit le narratif absent jusque-là du texte, de manière significative, avec douze occurrences, et une présentation de Williams comme homme du mystère ("Man of mystery », p. 131). La première conférence avait promis de rendre compte du cas John Williams, et c'est en relatant les étapes du meurtre, enfin présenté dans ce post-scriptum, que le texte retrouve l'idée du mystère dans sa quête des « hiéroglyphes » de l'affaire et l'évocation de leur caractère fascinant :

It is really wonderful and most interesting to pursue the successive steps of this monster, and to notice the absolute certainty with which the silent hieroglyphics of the case betray to us the whole process and movements of the bloody drama, not less surely and fully than if we had been ourselves hidden in Marr's shop, or had looked down from the heavens of mercy upon this hell-kite, that knew not what mercy meant ${ }^{14}$.

7 La quête de la vérité du crime n'est pas essentielle à son évaluation esthétique. Mais elle ne lui est pas absolument étrangère dans la mesure où elle permet à l'admirateur, par la reconstruction mentale, d'accéder à un mode de contemplation quasi-divin du spectacle impossible : l'aperception du crime entre alors en résonance avec l'esthétique sublime qui nourrit de fait en sous-main la pensée de De Quincey ${ }^{15}$, en lien étroit avec une approche cathartique de l'œuvre ${ }^{16}$. Le mystère est envisagé à la manière d'un effet artistique rehaussant le meurtre dans la mesure où il en augmente le potentiel de fascination ${ }^{17}$ et confère un aspect dynamique à cette recherche de la vérité du crime. On peut noter à cet égard que De Quincey réintroduit rétrospectivement, au moment de la publication en volume en 1854 , la mention du mystère dans la première conférence de 1827, de manière aussi ponctuelle qu'appuyée :

The finest work of the seventeenth century is, unquestionably, the murder of Sir Edmondbury Godfrey, which has my entire approbation. In the grand feature of mystery, which in some shape or other ought to colour every judicious attempt at murder, it is excellent; for the mystery is not yet dispersed. The attempt to fasten the murder upon the Papists, which would injure it as much as some well-known Correggios have been injured by the professional picture-cleaners, or would even ruin it by translating it into the spurious class of mere political or partisan murders, thoroughly wanting in the murderous animus, I exhort the society to discountenance. In fact, this notion is altogether baseless, and arose in pure Protestant fanaticism ${ }^{18}$.

8 Le mystère dans sa dimension herméneutique est considéré en tant qu'effet ; il concerne la puissance d'évocation du meurtre parce qu'il est, en lui, ce qui résiste au sens et suscite l'intérêt, hors de toute référence à l'horreur ou à la terreur - en quoi il échappe malgré tout au sublime ; la mention des catholiques comme faux auteurs du mystère fait, au passage, assurément sens sur le mode ironique en convoquant le souvenir des controverses théologiques ayant entouré le terme pour dire le jeu de manipulations abusives ${ }^{19}$. Mais ici, le mystère, dans sa dimension artificielle, n'est plus dévalorisé mais au contraire loué; il est ainsi en mesure d'exercer une action sur le spectateur stupéfait devant ce surgissement de l'inattendu. Dans l'essai «On the Knocking at the Gate in Macbeth ", De Quincey disait déjà la fascination pour l'impression produite par un signe imprévu entrant en résonance avec le meurtre et invitant le spectateur à reconsidérer ses conceptions initiales ${ }^{20}$. La thématisation esthétique du crime amène à investir le mystère à nouveaux frais, comme ce qui permet de produire sur le récepteur un effet spécifique et résolument original. 
9 Il se trouve donc que De Quincey a par ailleurs écrit un texte fictionnel préfigurant le genre policier. Il s'agit de "The Avenger " ${ }^{21}$, court récit publié en 1838 dans le Blackwood's Edinburgh Magazine $e^{22}$, et qui traite d'une série de meurtres d'allure inexplicable, qui touche de manière apparemment arbitraire différents habitants d'un village. À vrai dire, le titre et plus encore l'épigraphe orientent la lecture vers le thème d'une vengeance revêtant un caractère quasiment métaphysique : «Why callest thou me murderer, and not rather the wrath of God burning after the steps of the oppressor, and cleansing the earth when it is wet with blood?» $\left(\right.$ p. 35) ${ }^{23}$.

10 Le personnage principal, Maximilien Wyndham, qui occupe une place privilégiée dans le récit et surgit au moment où commencent les meurtres, est implicitement présenté d'emblée comme le tueur potentiel ; mais jusqu'au dénouement, le texte n'apporte pas au lecteur la certitude de sa culpabilité. Rien ne permet d'ailleurs, avant la confession finale, de comprendre quel mobile pourrait pousser cet étranger au meurtre. Évoquant des phénomènes terrifiants et des secrets inquiétants, le récit fait appel au mot " mystery ", qui apparaît vingt-deux fois sous ses diverses formes dans cette nouvelle pour dire le caractère troublant d'une logique criminelle effrénée à laquelle on ne comprend rien. Pour autant, le texte échappe largement à la tonalité gothique : si le récit traite bel et bien d'événements suscitant la terreur des villageois, il s'abstient de jouer de mécanismes d'identification propres à faire éprouver au lecteur l'effet de suspens ou de surprise. La narration du crime horrifique, objective et distanciée, engage ici prioritairement la curiosité du lecteur cherchant à comprendre ce dont il retourne : ce n'est pas le pronostic sur ce qui risque d'arriver, mais le diagnostic sur ce qui a bien pu se passer, qui constitue l'enjeu essentiel de la nouvelle ${ }^{24}$.

11 Le texte se présente comme un récit rétrospectif dont le narrateur anonyme est l'un des habitants du village où s'est déroulé le drame. Il connaît l'ensemble des protagonistes et a logé Maximilien Wyndham, mais adopte un point de vue largement extérieur aux événements racontés. Il ne laisse deviner qu'à la fin du texte qu'il avait soupçonné Maximilien, comme le lecteur lui-même aux yeux duquel ce seul personnage a pris consistance au cours de la narration ${ }^{25}$; l'un des seuls moments où le narrateur joue un rôle effectif dans le récit correspond à un épisode où il se situe encore du côté du regard. Entrant dans la chambre de Maximilien, il contemple son visage qui lui rappelle quelqu'un, puis regarde le médaillon qu'il tient en main. Le narrateur cherche à décoder et saisir des signes de ce qui se présente, et met le lecteur en position d'observateur cherchant à ordonner une suite incompréhensible d'événements.

De la sorte, le récit présente de manière distanciée les événements passés entourant les meurtres. Ceux-ci, multipliés comme sans raison, touchant des personnages de tous âges et conditions, ont pourtant semblé aux villageois mystérieux et terrifiants :

I was present from first to last, and watched the whole course of the mysterious storm which fell upon our devoted city in a strength like that of a West Indian hurricane, and which did seriously threaten at one time to depopulate our university, through the dark suspicions which settled upon its members, and the natural reaction of generous indignation in repelling them (p. 35 ${ }^{26}$.

13 Le texte évoque une logique de la terreur face au meurtre incompréhensible ouvrant à une déstabilisation de la collectivité. Cette terreur dérive d'une incapacité à donner sens aux événements, qui équivaut à une perte de maîtrise dans le droit fil de la tradition gothique :

The more certain did these arrangements strike people as remedies for the evil, so much the more effectually did they aid the terror, but, above all, the awe, the sense 
of mystery, when ten cases of total extermination, applied to separate households, had occurred, in every one of which these precautionary aids had failed to yield the slightest assistance (p. 36) ${ }^{27}$. d'ouvrir sur une réalité radicalement inconnue, et propre dès lors à ouvrir à tous les fantasmes :

The general feeling [...] was, even among the boldest, like that which sometimes takes possession of the mind in dreams-when one feels oneself sleeping alone, utterly divided from all call or hearing of friends, doors open that should be shut, or unlocked that should be triply secured, the very walls gone, barriers swallowed up by unknown abysses, nothing around one but frail curtains, and a world of illimitable night, whisperings at a distance, correspondence going on between darkness and darkness, like one deep calling to another, and the dreamer's own heart the center from which the whole network of this unimaginable chaos radiates, by means of which the blank privations of silence and darkness become powers the most positive and awful (p. 36-37) ${ }^{28}$.

manque à comprendre, lié à la dynamique du mystère, engage les villageois à modifier leur regard sur le monde. Parce qu'ils ne comprennent pas les phénomènes qui surgissent autour d'eux, ils ne peuvent que tendre à y voir l'exercice d'une force supérieure. Le sublime burkéen, et le souvenir de la conception théologique du mystère sacré comme manifestation insaisissable de la transcendance, travaillent un blocage du sens qui se charge de tout le potentiel de menace dont le mot est porteur. Mais on l'a dit, ce récit met précisément à distance l'effet de terreur qu'il présente au lecteur par le biais de son dispositif narratif fondé sur une position d'extériorité.

fisant narration d'une terreur collective, mais sans inviter le lecteur à y participer, le texte invite en réalité ce dernier à s'engager dans une quête de compréhension : il ne s'agit pas d'éprouver l'effet de terreur, mais de saisir ce qui a bien pu le produire, d'appréhender les mécaniques secrètes qui ont conduit la collectivité au vertige de l'effroi. Tout se passe comme si le récit mettait en scène l'effet gothique pour mieux le mettre à distance et engager une lecture au second degré destinée à en déconstruire les effets. Aussi met-il en place, face aux événements terrifiants, un autre régime du mystère attaché à Maximilien, et qui relève de la logique de l'incompréhensible ; le texte propose une équation entre Maximilien et l'idée de mystère («mysterious Maximilian », p. 41) dans la mesure où ce personnage concentre en lui les contrastes, et incarne de la sorte un rapport à un secret enfoui qui seul permettrait de donner sens aux contradictions dont il semble pétri. Jeune homme doué au plus haut degré de toutes les qualités, mais lourd de mélancolie, Wyndham est rabattu du côté du sens incertain. Il éveille moins la terreur que la perplexité du fait qu'il sort à tous égards de la norme :

This result was not a pure effect of manly beauty, however heroic, and in whatever excess; it arose in part from the many and extraordinary endowments which had centered in his person, not less from fortune than from nature; in part also, as I have said, from the profound sadness and freezing gravity of Mr. Wyndham's manner; but still more from the perplexing mystery which surrounded that sadness (p. 41) ${ }^{29}$.

Homme du secret, concentrant en lui le blocage de la signification, Maximilien renvoie à une version alternative du mystère, susceptible de supplanter l'approche gothique de ce dernier en ce qu'il ouvre bel et bien désormais à la stimulation de la quête du sens en et pour lui-même : il ne s'agit plus d'éprouver l'effroi de quelque réalité d'apparence 


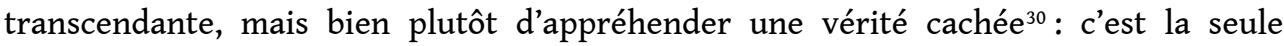
curiosité du lecteur que le récit cherche avant tout à susciter. Mais pour ce faire, encore faut-il donner à penser que quelque sens profond et inaperçu pourrait se donner à lire dans ses lignes. Maximilien donne consistance à cette figure du manque et du trouble dans le sens dans la mesure où il est porteur d'un secret que le lecteur n'est pas en mesure de percer. Il est vrai qu'au terme du récit surviennent enfin ses aveux écrits, qui prennent la forme d'une révélation; il ne s'agissait au fond que d'une vengeance. Mais De Quincey, comme Poe, aime à compliquer la lecture de ses récits en y intégrant des effets d'ambiguïté à même d'en complexifier la lecture.

18 La confession de Maximilien, telle qu'elle nous est donnée à lire par le narrateur, est ainsi à certains égards suspecte, dans la mesure où elle est assortie d'un testament sous conditions, fait au bénéfice de ce même narrateur. Maximilien lui a légué sa fortune, à condition qu'il garde le secret pendant trois ans sur la vérité des meurtres. Se pose alors la question de la crédibilité d'une voix énonciative apparemment extérieure au récit mais en réalité profondément intéressée, puisqu'elle appartient au bénéficiaire indirect des meurtres, dont le statut est dès lors peu ou prou celui d'un complice. De la sorte, la curiosité du lecteur est à la fois stimulée et frustrée. Le trouble herméneutique, au moment de se voir apparemment comblé, suscite une interrogation portant cette fois sur le statut même du récit qui nous est rapporté, d'autant que si Maximilien est mystérieux, c'est aussi en tant qu'écrivain ${ }^{31}$, comme le dit le texte au moment d'introduire la phase de révélation : «Much of this WOULD be unveiled -all might be: and there and then, with the corpse lying beside me of the gifted and mysterious writer, I seated myself, and read the following statement [...]» (p. 68 $)^{32}$. Doué et mystérieux, écrivain et cadavre, tel est Maximilien au terme du récit, alors qu'il y joue aussi fondamentalement le rôle de l'assassin. Il est à la fois la figure de l'incompréhensible et l'ordonnateur du trouble dans le sens.

On voit comment le récit du crime engage puissamment le désir de lecture en convoquant l'idée d'un sens résolument instable. La nouvelle mobilise deux régimes de fonctionnement du mystère, en relation l'un avec l'autre tout en relevant de modalités de fonctionnement différentes. D'un côté, il y a le mystère auquel sont confrontés les villageois, et qui les plonge dans la terreur à la manière de victimes d'un récit gothique démunies face à une force hostile et insituable; et de l'autre, il y a le mystère qui concerne la mise en place de ce mystère premier; il concerne un secret à connaître, et engage un processus de quête herméneutique, auquel le lecteur est invité à participer alors même qu'il serait bien incapable d'en saisir les tenants et les aboutissants, puisque ce secret renvoie à l'art que Maximilien et le narrateur ont déployé tour à tour pour produire un texte fondamentalement ambigu. Récit protopolicier, si l'on veut, "The Avenger " nous dit bien qu'il y a quelque chose à connaître, qu'il nous faut chercher, et que pourtant nous ne trouverons pas par nous-mêmes.

Mais dans cette économie textuelle, pour que fonctionne l'appel au "mystère », que la fascination du lecteur soit suffisamment mobilisée, l'idée d'un sens profond, transcendant peut-être, se trouve également convoquée, en lien avec les origines théologiques du terme. Le récit joue avec l'idée d'autres plans de signification, et courtcircuite ainsi l'idée d'une rupture radicale entre un pur rapport à la curiosité du lecteur et ce qui engagerait une portée plus profonde, dans la mesure même où il invite à interpréter le texte pour en déployer les possibles, conformément à la leçon patristique invitant à chercher les différents sens de l'Écriture. Si le meurtre se fait le moyen non 
plus de dire la seule terreur, mais la quête herméneutique comme telle dans son rapport au processus de lecture, c'est alors en lien discret avec des thématiques religieuses que la nouvelle donne à lire en sous-main.

21 De fait, si le scandale moral est présent dans le texte, il concerne en réalité une forme de meurtre autre que celle qui a nourri le récit : la révélation finale est celle d'un péché originel, d'un crime profond et enfoui engageant la communauté villageoise à l'égard de la mère juive du héros. Cette dernière est d'ailleurs présentée dans le récit comme une descendante des Maccabées $^{33}$, que la tradition chrétienne considérait selon la lecture typologique comme préfigurant les martyrs chrétiens ${ }^{34}$. Les assassinats perpétrés par Maximilien ont valeur de châtiment et témoignent d'une culpabilité première de la collectivité. Ils revêtent dès lors un statut moral essentiellement ambigu. La tradition théologique $\mathrm{du}$ mystère, qui engageait un rapport à l'herméneutique textuelle, permet de mettre en relation le motif du meurtre et une approche du récit envisagé comme espace de questionnement, et elle invite à revisiter le motif d'une culpabilité fondamentale de l'humanité, de réalités ordinaires derrière lesquelles se lit l'ombre du mal humain dans sa dimension métaphysique, et susceptible de concerner aussi le lecteur. Ce dernier, curieux et déstabilisé face au récit de ces crimes mystérieux, se trouve après tout dans une posture analogue à celle de ces villageois inconscients du mal dont ils sont porteurs.

Ce que dispose, par le biais du meurtre insituable et ambigu, ce texte de 1838 de De Quincey, c'est une mise en scène du mystère terrifiant, mais aussi sa dévaluation au profit de cet autre régime du mystère qui concerne le sens incertain de l'histoire présentée au lecteur, et se voit lui-même connecté à la tradition de l'herméneutique biblique. Se donne à lire ici un traitement du récit du crime qui en déploie le potentiel de fascination, et joue du rapport à la terreur aussi bien que de la pure curiosité, mais au bénéfice de cette dernière, qui finit par l'emporter résolument.

Par ailleurs, si la quête herméneutique fonctionne en et pour elle-même, elle pourrait bien trouver sa pertinence, on le voit, de plonger dans des racines plus profondes. Ce que le texte invite alors peut-être déjà à explorer, ce sont les raisons mêmes du pouvoir du récit de mystère, en amenant le lecteur à s'interroger sur les raisons qui le poussent à céder à une curiosité qui pourrait bien avoir elle-même à voir avec quelque culpabilité première... Euvre de transition, adieu au genre gothique, ou encore récit policier avant la lettre relevant de quelque dandysme littéraire ? Si l'on tient à adopter un regard ouvertement rétrospectif sur le texte, sans doute est-il loisible d'envisager ainsi " The Avenger »; mais cette nouvelle se fait aussi, et surtout, réflexion au second degré sur les mécanismes qui confèrent au récit son emprise sur le lecteur. Le " mystère », entre dispositif immersif et rapport au sens, parle peut-être ici de la sorte d'un moment particulier de l'histoire littéraire, en lien avec l'émergence de la culture médiatique, dans une fiction qui rêve de jouer simultanément sur une multitude de tableaux, et de susciter le désir de lecture tout en plongeant dans le vertige des possibles du texte... 


\section{NOTES}

1. Voir par exemple Dominique Kalifa, Crime et culture au XIXe siècle, Paris, Perrin, 2005.

2. Rappelons que le "mystère ", progressivement rattaché en contexte catholique à un rapport au sacré, à une transcendance radicale exigeant du croyant un acte de foi inconditionnel, et à des aspects du culte illustrant le caractère incompréhensible de la divinité, a constitué une cible privilégiée pour le protestantisme, qui y a vu dans une large mesure l'expression du désir de l'institution papale d'asseoir son pouvoir sur les fidèles. La « terreur sacrée » dont est porteur le mystère relève selon cette perspective du faux, de la stratégie de manipulation, ou, si son pouvoir est reconnu, il est alors associé à l'exercice du démon (sur ce sujet, voir par exemple Denis Crouzet, Les Guerriers de Dieu. La violence au temps des troubles de religion, vers 1525-vers 1610, Seyssel, Champ Vallon, 1990, 2 volumes ; Claude Postel, Traité des invectives au temps de la Réforme, Paris, Les Belles Lettres, 2004). Ces questions ont d'ailleurs pu ouvrir sur des considérations engageant la littérature (Baudouin Millet, "Ceci n'est pas un roman ». L'évolution du statut de la fiction en Angleterre de 1652 à 1754, Louvain/Paris/Dudley, Peeters, 2007) et le rapport au travail du style, aisément rattaché à une artificialité suspecte. Le « mystère ", progressivement laïcisé, s'est ainsi chargé de connotations sémantiques qui en ont fait un terme essentiellement ambivalent, entre vérité et mensonge, rapport à la divinité ou à l'instance diabolique, jeu de manipulation ou accès effectif à un rapport au monde irréductible au jeu de la raison, ce qui lui a conféré son efficace particulière. Pour une analyse détaillée de ces enjeux, nous nous permettons de renvoyer à la première partie de notre thèse, "Mystère et jouissance narrative: enjeux de dramatisation dans la fiction policière émergente », sous la direction d'Arnaud Huftier, Université Polytechnique Hautsde-France, soutenue le 22 octobre 2020 (à paraître).

3. On peut notamment lire la nouvelle d'Edgar Poe, "The Murders in the Rue Morgue ", dans une telle perspective: le texte convoque de fait l'imaginaire augustinien d'une perte de sens découlant de la faute originelle, qu'il met concrètement en scène avec ce gorille rejouant l'entrée dans l'humanité par le biais de l'accès au crime. Le propos convoque alors discrètement un fonds théologique pour rendre compte de l'inaptitude humaine à saisir la vérité, tout en s'articulant à une approche textuelle fondée sur les jeux polysémiques et destinée à susciter la curiosité du lecteur. Sur cette question, Marc Vervel; "Mystère et ombres religieuses : une proposition de relecture des enjeux herméneutiques dans "Murders in the Rue Morgue" d'Edgar Allan Poe ", Revue française d'études américaines, à paraître fin 2021.

4. Barbara M. Benedict, Curiosity. A Cultural History of Early Modern Inquiry, Chicago/Londres, The University of Chicago Press, 2001. L'auteur voit l'affirmation progressive d'une véritable épistémè de la curiosité entre 1660 et 1820 , en lien avec le rêve d'une conquête symbolique du monde, notamment emblématisée par la figure de Robinson : "Robinson Crusoe incarnates the cultural ideal of transcendent curiosity. Even more than Defoe's other explorers, he escapes time and space; even more than other conquerors, he emblematizes cultural ambition ", p. 109.

5. Michel Foucault, Surveiller et punir (1975), Paris, Gallimard, « Tel », 2005.

6. Ibid., p. 82-83.

7. Thomas de Quincey, Euvres, Paris, Gallimard, « Pléiade », 2011, p. XLVI ; Patrick Bridgwater, De Quincey's Gothic Masquerade, Amsterdam/New York, Rodopi, 2004, «Early Gothic reading », p. 11-27.

8. À commencer par le roman Klosterheim, publié en 1832. Pour Patrick Bridgwater, De Quincey peut de manière bien plus profonde être considéré comme un auteur vraiment "gothique»; mais par ce terme, il fait à vrai dire moins référence au genre gothique, en tout cas en son sens historique étroit (tel que l'appréhende par exemple Maurice Lévy dans son ouvrage classique, Le Roman " gothique » anglais, 1764-1824 (1968), Paris, Albin Michel, 1995) que d'une manière élargie, 
comme ce qui relève d'une expression des troubles de la conscience et d'une intériorité tourmentée («[...] haunted consciousness and, more generally, [...] interiority, interior space », Patrick Bridgwater, De Quincey's Gothic Masquerade, op. cit, p. 65 ; voir l'ensemble du chapitre « De Quincey and Gothic », p. 60-79).

9. Rappelons que cette revue conservatrice publie par ailleurs aussi bien des productions des lake poets que des textes d'inspiration sensationnaliste. Elle est notamment célèbre pour ses récits à tonalité gothique, signés aussi bien de James Hogg que de Henry Thomson ou William Godwin Jr. Parmi les cibles de la nouvelle de Poe publiée en 1838, "How to write a Blackwood article", figure notamment De Quincey, les Confessions d'un fumeur d'opium anglais se trouvant nommément cité (alors que ce texte ne fut pas en réalité publié dans le Blackwood's Magazine; sur cette question, voir Thomas de Quincey, Euvres, op. cit., p. 1591). Pour un échantillon en français des nouvelles à tonalité gothique publiées dans la revue, André Fayot (éd.), Le Revenant et autres contes de terreur du Blackwood Magazine, Paris, José Corti, « Domaine romantique », 2000.

10. Sur la prolixité d'une œuvre qui aborde toutes sortes de sujets et les envisage selon une multitude d'approches textuelles, Josephine McDonagh, De Quincey's Disciplines, Oxford, Clarendon Press, 1994. Sur le rapport à l'entre-deux d'une œuvre érudite et traversée d'incertitudes, Céline Lochot, "Thomas De Quincey between Romantics and Victorians ", Cahiers victoriens et édouardiens, 87/2018. En ligne: https://journals.openedition.org/cve/3641. Consulté le 15/03/2021.

11. Thomas de Quincey, On Murder Considered as One of the Fine Arts, dans On Murder, Oxford, Oxford University Press, "Oxford World's Classics», 2006, p. 8-34 ; l'édition reprend la version originale des deux mémoires et du post-scriptum composant le texte, qui ont été respectivement publiés en 1827, 1839 et 1854. Pour la version révisée, publiée en 1854 à l'occasion de l'ajout du post-scriptum, voir De Quincey's Works, vol. IV, The English-Mail Coach and Other Writings, Edinburgh, Adam and Charles Black, 1863, p. 1-110. Pour la version française, on se reportera à De l'assassinat considéré comme un des Beaux-Arts dans Thomas de Quincey, Euvres, op. cit., p. 1235-1335.

12. C'est bien sûr dans le Blackwood's Magazine, notamment connu comme on l'a dit pour ses récits à tonalité sensationnaliste, que cette publication a eu lieu. De Quincey, qui vécut largement de ses contributions aux journaux, fut un collaborateur régulier de la revue.

13. "The Society of Connoisseurs in Murder ", dans Thomas de Quincey, On Murder, op. cit., p. 8.

14. Ibid., p. 107. «Il est vraiment merveilleux, et du plus grand intérêt, que de suivre, les uns après les autres, les pas de ce monstre, et d'observer avec quelle certitude absolue les hiéroglyphes silencieux de l'affaire trahissent tout le processus et chacun des mouvements du drame sanglant - non moins sûrement, non moins pleinement que si nous avions été cachés nous-mêmes dans la boutique de Marr ou que si nous avions contemplé du haut des Cieux miséricordieux ce vautour de l'enfer qui ne savait point ce que la miséricorde voulait dire ", Cuvres, op. cit., p. 1298.

15. La question du rapport de De Quincey au sublime a été largement analysée ; voir par exemple Charles J. Repzka, Sacramental Commodities. Gift, Text, and the Sublime in De Quincey, Amherst, University of Massachusetts press, 1995. Le texte développe l'idée que, dans une époque de marchandisation du livre, De Quincey reconfigure l'esthétique du sublime de manière à faire du livre un lieu de communion spirituelle, porteur de ce fait d'un effet spécifique sur le lecteur.

16. Le texte noue ainsi pensée du rapport de l'art à la violence, approche kantienne de la contemplation esthétique, théorie burkéenne du sublime et pensée aristotélicienne de la « catharsis »; voir la notice de l'édition « Pléiade », p. 1734.

17. "One murder is compared with another; and the circumstances of superiority, as, for example, in the incidence and effects of surprise, of mystery, etc., are collated and appraised ", Thomas de Quincey, On Murder, op. cit., p. 97. 
18. De Quincey's Works, vol. IV, op. cit., p. 26-27. "Le plus bel ouvrage du XVII ${ }^{\mathrm{e}}$ siècle est sans conteste l'assassinat de sir Edmundbury Godfrey, qui a toute mon approbation. Pour le trait grandiose du mystère, qui, sous une forme ou sous une autre, doit venir rehausser toute judicieuse tentative d'assassinat, il excelle ; car le mystère n'est pas encore dissipé. On a tenté d'attribuer ce meurtre aux papistes, ce qui serait lui faire injure autant que les nettoyeurs de tableaux professionnels ont fait injure à certains Corrège bien connus, ou même le ruiner en le transférant dans la catégorie bâtarde des simples meurtres politiques ou partisans, auxquels fait entièrement défaut l'animus meurtrier, et j'exhorte l'assistance à réprouver le fait. À vrai dire, c'est là une notion dépourvue de tout fondement, née purement et simplement du fanatisme protestant ", Euvres, op. cit. p. 1257-1258.

19. Il faut bien sûr se rappeler à cet égard que le protestantisme s'est largement institué en critique du «mystère » catholique, vu dans ses dimensions doctrinales et liturgiques comme un obscurcissement de la parole divine produit à des fins de manipulation ; voir par exemple claude Postel, Traité des invectives au temps de la Réforme, Paris, Les Belles Lettres, 2004. Les polémiques à propos de la religion naturelle, à l'époque des Lumières, sont en large partie un avatar de ces querelles. Brian Walter Young, Religion and Enlightenment in Eighteenth-Century England. Theological Debate from Locke to Burke, Oxford/New York, Clarendon Press/Oxford University Press, 1998.

20. "On the Knocking at the Gate in Macbeth», dans Thomas de Quincey, On Murder, op. cit., p. 3-7; « Du heurt à la porte dans Macbeth », p. 1227-1234 de l'édition française.

21. "The Avenger», dans Thomas de Quincey, On Murder, op. cit., p.35-80; «Le Bras de la vengeance », p. 1537-1588 de l'édition française des Æuvres en Pléiade.

22. Le texte initialement proposé à l'éditeur était nettement plus long mais fut largement amputé avant publication ; voir à ce propos la notice de l'édition française, p. 1783.

23. «Pourquoi voir en moi un criminel et non la colère de Dieu qui poursuit ardemment les pas de l'oppresseur et purifie la terre quand elle est marquée de sang? ", CEuvres, op. cit., p. 1537. Il s'agit là d'une citation fictive, le texte étant selon toute probabilité de De Quincey. Voir à ce sujet la note 1 de l'édition « Pléiade », ibid., p. 1786.

24. Nous reprenons bien évidemment ici la terminologie utilisée par Raphaël Baroni dans La Tension narrative : suspense, curiosité et surprise, Paris, Seuil, 2007.

25. Thomas de Quincey, "The Avenger », op. cit., p. 68.

26. "J'ai été présent du début à la fin et j'ai suivi, dans tout son déroulement, cette mystérieuse tempête qui s'est abattue sur notre chère ville avec la violence d'un ouragan des Antilles et qui, à un moment donné, a même menacé sérieusement de dépeupler notre université, à cause des noirs soupçons qui se sont portés sur ses membres, ainsi que la réaction naturelle de généreuse indignation qui les a repoussés ", Euvres, op. cit. p. 1539-1540.

27. «Plus les dispositions pour remédier au mal paraissaient sûres, plus elles favorisaient efficacement la terreur, mais surtout l'effroi - le sentiment d'un mystère, lorsque dix cas d'extermination se produisirent dans des foyers distincts, où chaque fois, les précautions prises avaient échoué à apporter la moindre aide », ibid., p. 1540.

28. «Le sentiment public [...] ressemblait, même chez les plus courageux, à celui qui parfois s'empare de l'esprit pendant les rêves - quand vous vous sentez seul dans le sommeil, complètement isolé des amis que vous ne pouvez pas appeler, qui ne peuvent vous entendre, alors s'ouvrent des portes qui devraient être fermées, ou bien est déverrouillé ce qui devrait être bouclé à triple tour ; les murs eux-mêmes ont disparu ; des barrières ont été englouties dans des abîmes inconnus ; il ne reste rien autour de vous, sinon des rideaux légers ; un monde de nuit sans fin; des chuchotements au loin; l'obscurité qui répond à l'obscurité comme un gouffre en appelle un autre, tandis que le corps du rêveur forme le centre d'où rayonne tout le réseau de cet inimaginable chaos, par lequel les simples privations du silence et de l'obscurité deviennent des forces très positives et redoutables ", ibid., p.1541. Ce passage évoque de manière directe la 
puissance de la nuit en lien avec la conception burkéenne du sublime : sur cette question, Max Milner, L'Envers du visible. Essai sur l'ombre, Paris, Éditions du Seuil, 2005, p. 152-156.

29. « Assurément, cela ne tenait pas uniquement à l'effet produit par sa mâle beauté, si héroïque et excessive; cela provenait en partie des dons nombreux et extraordinaires qui avaient été accumulés en sa personne aussi bien par la fortune que par la nature ; en partie aussi, comme je l'ai dit, de la tristesse profonde et de la gravité réfrigérante du comportement de M. Wyndham, mais plus encore du mystère intrigant qui entourait cette tristesse », CEuvres, op. cit., p. 1545-1546. 30. "A person so mysterious took precedency of other interests even at a time like this", Thomas de Quincey, On Murder, op. cit., p. 50 ; «Une personnalité aussi mystérieuse éclipsait tout le reste, même dans un moment pareil », Oeuvres, op. cit., p. 1556.

31. Proposition qui découle de l'équivalence entre artiste et meurtrier construite dans l'œuvre de De Quincey. A. S. Plumtree, «The Artist as Murderer: De Quincey's Essay on Murder Considered as One of the Fine Arts, dans Robert Lance Snyder (sld.), Thomas de Quincey. Bicentenary Studies, Norman, University of Oklahoma Press, 1985, p. 140-163.

32. «Beaucoup de tout cela serait sans doute révélé - peut-être bien tout ; c'est donc à cet instant et en ce lieu, près du corps de celui qui l'avait rédigé, un jeune homme mystérieux et comblé de dons, que je m'assois pour lire la déclaration suivante [...]», Euvres, op. cit., p. 1576. La formule «mysterious writer» permet d'associer la figure de l'assassin à celle de l'écrivain, l'un valant pour l'autre dans la mesure où ils sont tous deux les orchestrateurs du mystère.

33. Thomas de Quincey, « The Avenger », op. cit., p. 70.

34. Voir par exemple, dans Marie-Françoise Baslez, Olivier Munnich (sld.), La Mémoire des persécutions. Autour des livres des Maccabées, Paris/Louvain, Peeters, 2014, les articles de Gérard Nauroy, « Du héros juif au saint chrétien : l'appropriation du martyre des frères Maccabées par Ambroise de Milan », p. 347-370, et de Régis Courtray, «Les Maccabées dans l'œuvre de Jérôme : de la libération juive à la victoire dans le Christ», p. 385-398 ; et Raphaëlle Zadé, Les martyrs Maccabée : de l'histoire juive au culte chrétien. Les homélies de Grégoire de Nazianze et de Jean Chrysostome, Leiden/Boston, Brill, 2007.

\section{RÉSUMÉS}

Cet article se penche sur la manière dont De Quincey appréhende la question de l'élucidation criminelle. Associé à une approche esthétisante du crime, il envisage en effet sous cet angle la question du "mystère", vu comme susceptible de produire un effet propre dans On Murder Considered as One of the Fine Arts. Avec "The Avenger ", il propose d'ailleurs déjà en 1838, avant donc que Poe ne crée le personnage de Dupin, une fiction criminelle invitant le lecteur à se mettre en quête du sens évanescent. Ici se lit un pacte de lecture anticipant à certains égards celui du genre policier, mais qui se fonde sur un radical vertige herméneutique, où le trouble dans la signification est aussi travail de références religieuses pour donner consistance au traitement narratif. Le mystère, entre visée narratologique et convocation discrète de résonances théologiques, se constitue en interface littéraire pour confronter le lecteur au sens insaisissable.

\section{INDEX}

Mots-clés : mystère, récit criminel, récit policier, curiosité, De Quincey 


\section{AUTEUR}

\section{MARC VERVEL}

Marc Vervel est un ancien étudiant de l'Ecole Normale Supérieure LSH, agrégé de I'université (lettres modernes), docteur en littérature, enseignant à l'ufr LAC (Lettres, Arts, Cinéma) de l'Université de Paris. Sa thèse, soutenue en octobre 2020, s'intitule : «Mystère et jouissance narrative. Enjeux de dramatisation dans la fiction policière émergente » (direction Arnaud Huftier, De Scripto, Université Polytechnique Hauts de France ; à paraître).

Laboratoire de rattachement : Cerilac, Université de Paris. Chercheur associé à DeScripto, Université Polytechnique Hauts-de-France.

Dernières publications :

«De quand date le "roman policier" »?, Le Rocambole, n95-96, été-automne 2021, p. 273-278.

« L'Espion de police de Lamothe-Langon : roman policier, roman de mœurs, roman populaire ? », Le Rocambole, n`93-94, hiver 2020-printemps 2021 , p. 253-262.

« L'ombre du surnaturel, du gothique au récit policier », revue Chameaux, université de Laval, Québec, $n^{\circ} 11$, mars 2019, « Magie, sorcellerie et surnaturel en littérature ».

« L'affaire Lindbergh et Le Crime de l'Orient Express : enjeux de la reconfiguration narrative en régime fictionnel d'un fait drivers en manque de clôture », revue Intercâmbio, Université de Porto, 2e série, vol. 11, 2018, p. 43-62.

marc.vervel@u-paris.fr 